\title{
Correlation of Sympathovagal Imbalance with Disease Activity and Inflammatory Markers in South Indian Patients with Rheumatoid Arthritis
}

\author{
Selvakumar Ganesan ${ }^{1}$, Vir Singh Negi ${ }^{2}$, Girwar Singh Gaur, ${ }^{1, *}$, Vivek Kumar Sharma ${ }^{3}$, Gopal Krushna Pal ${ }^{1}$
}

\section{Selvakumar Ganesan", Vir Singh Negi'2, Girwar Singh Gaur ${ }^{1, *}$, Vivek Kumar Sharma ${ }^{3}$, Gopal Krushna $\mathrm{Pal}^{4}$}

'Department of Physiology, Jawaharlal Institute of Post Graduate Medical education and Research, Puducherry, INDIA.

${ }^{2}$ Department of Clinical Immunology Jawaharlal Institute of Post Graduate Medical education and Research. Puducherry, INDIA.

${ }^{3}$ Department of Physiology,

Government Institute of Medical Sciences, Kasna, Greater Noida, Uttar Pradesh, INDIA.

\section{*Correspondence}

\section{Dr. Girwar Singh Gaur}

Professor, Department of Physiology, Jawaharlal Institute of Post Graduate Medical education and Research, Puducherry-605006, INDIA.

Phone: +91 9994470395

Email: drgsgaur@yahoo.com

History

- Submission Date: 08-04-2020;

- Review completed: 16-05-2020;

- Accepted Date: 10-06-2020.

DOI : 10.5530/ijcep.2020.7.2.15

\section{Article Available online}

http://www.ijcep.org

\section{Copyright}

(C) 2020 Phcog.Net. This is an openaccess article distributed under the terms of the Creative Commons Attribution 4.0 International license.

\begin{abstract}
Background and Aim: Rheumatoid arthritis (RA) patients have higher risk of cardiovascular disease and mortality and that is not linked to traditional cardiovascular risk factors. Altered sympathovagal balance is associated with cardiovascular disease. In RA sympathovagal balance and inflammatory markers were reported to be increased, but there is inadequacy of data on its assessment and association with disease activity. This study was conducted to find the correlation of sympathovagal balance with disease activity and inflammatory markers in South Indian patients with RA. Methods: RA patients (diagnosis of RA made as per 2010 ACR/EULAR classification criteria) were enrolled from Department of Clinical Immunology, JIPMER. Participants were sub grouped as low disease activity (LDA), moderate disease activity (MDA) and severe disease activity (HDA) using disease activity score 28 with ESR. Frequency and time domain parameters of heart rate variability and inflammatory markers like Interleukin-1 $\alpha$ (IL-1 $\alpha$ ), Interleukin-6 (IL-6), tumor necrosis factor- $\alpha$ (TNF $\alpha$ ) and cortisol were measured for all the participants. Results: A total of 143 RA patients (23 in LDA group, 69 in MDA group and 51 in HDA group) were assessed during the study. LF-HF ratio (ratio of low frequency power to high frequency power) a marker of sympathovagal balance showed significant increase in MDA and HDA group compared to LDA group. LF-HF ratio correlated positively with DAS28 ( $r=0.473, p<0.001)$, cortisol $(r=0.363, p<0.001)$, IL $-1 \alpha(r=0.379$, $p<0.05)$. Conclusion: Sympathovagal imbalance was significantly increased in the MDA and HDA group compared to LDA group and positively correlated with disease activity, IL-1 $\alpha$ and cortisol in the patients of RA. Assessment of sympathovagal balance may help to find the autonomic dysfunction and cardiovascular risk in RA.

Key words: Autonomic dysfunction, Cardiovascular risk, HRV, Inflammatory markers, Rheumatoid arthritis, Sympathovagal balance.
\end{abstract}

\section{INTRODUCTION}

Rheumatoid arthritis (RA) is an autoimmune inflammatory rheumatic disease. It involves the joints symmetrically and causes inflammation mediated damage. The disease also affects various organ systems causing cardiovascular, pulmonary, skin and ocular involvement. ${ }^{[1,2]}$ Accumulating evidence suggests that chronic inflammatory nature of RA leads to the development of cardiovascular complications. ${ }^{[3,4]}$ RA patients have higher risk of developing myocardial infarction, heart failure and cerebrovascular disease than the general population. ${ }^{[5-7]}$ Traditional cardiovascular risk (CV) factors were not associated with higher cardiovascular complications in RA. ${ }^{[8,9]}$

Heart rate variability (HRV) is the variation of the time intervals between adjacent heartbeats. Assessment of HRV is a noninvasive method to measure various reflex autonomic changes that occur in the cardiac autonomic system. ${ }^{[10]}$ Assessment of sympathetic and parasympathetic

activity of autonomic nervous system has been accepted as a good predictor of cardiovascular risk. Important component of frequency domain analysis of HRV is LF-HF ratio (ratio of low frequency power which indicates sympathetic activity to high frequency power which indicates parasympathetic activity), a marker of sympathovagal balance. ${ }^{[11,12]}$ Sympathovagal balance is the balance of autonomic functions of an individual at any time. Nerve supply from sympathetic and parasympathetic system regulate important functions of the body like circulatory, immune, excretory, reproductive, respiratory systems and metabolism. ${ }^{[13]}$ Limited number of studies in RA have reported that high activity of sympathetic component leads to ventricular tachyarrhythmia and sudden death and reduced HRV in patients with heart disease is linked to elevated risk of CV incidents. ${ }^{[14,15]}$

Increased levels of interleukin-6 (IL-6), tumor necrosis factor- $\alpha(\mathrm{TNF} \alpha)$ and acute-phase reactants

Cite this article: Ganesan S, Negi VS, Gaur GS, Sharma VK, Pal GK. Correlation of Sympathovagal Imbalance with Disease Activity and Inflammatory Markers in South Indian Patients with Rheumatoid Arthritis. Int J Clin Exp Physiol. 2020;7(2):58-62. 
causes endothelial activation which alter the stability of atheromatous plaques and lead to higher CV risk in RA patients. ${ }^{[16]}$ Cytokines like interleukin-1(IL-1), IL-6 and TNF- $\alpha$ released from inflamed joints increase sympathetic activation and affect myocardium. ${ }^{[1]}$ Cytokines also affect the potassium and calcium channels causing extended action potential. ${ }^{[18-20]}$ Studies are limited in RA assessing the relationship between disease activity, HRV and inflammatory markers. ${ }^{[21]}$ Therefore, this study was planned to find the correlation of sympathovagal balance with disease activity and inflammatory markers in South Indian patients with newly diagnosed RA.

\section{MATERIALS AND METHODS}

This was a cross-sectional study conducted at the Department of Physiology in collaboration with Department of Clinical Immunology JIPMER. It was duly approved by the institute ethics committee (Reg. no. JIP/IEC/2015/14/554) and carried out between July 2015 to June 2018.

\section{Participants}

The diagnosis of RA was made according to the 2010 ACR / EULAR rheumatoid arthritis classification criteria. ${ }^{[22]} \mathrm{RA}$ patients attending the outpatient clinic of the Department of Clinical Immunology, JIPMER were screened for this study. All participants were explained about the study in their native language (Tamil). They were explained the study procedure, likely benefits and harms and those willing to participate were enrolled after obtaining written informed consent. The patients were classified as having low, moderate and high disease activity as per disease activity score 28 criteria. The study participants included both genders, between 30 to 60 years of age, who were on a stable dose of disease modifying antirheumatic drugs (single or combination therapy) in the last 3 months were included in the study. ${ }^{[23]}$ RA patients with history of comorbidities like diabetes mellitus, uncontrolled hypertension, any other neuromuscular, auto immune disorder, on yoga therapy or any other bio-feedback techniques and history of alcoholism or drug abuse were excluded.

After the recruitment and collection of baseline information related to disease activity, damage etc. Disease activity of all the participants was calculated with the help of disease activity score 28 using ESR (DAS28ESR) by an independent treating physician not a part of the study team. Cardiovascular parameters were evaluated in the autonomic research laboratory of the Department of Physiology.

\section{Sub Grouping of Participants}

Using Disease Activity Score in 28 Joints (DAS28ESR), we had divided the participants into the following three groups:

Low disease activity (LDA) group: DAS28 $>2.6$ to $\leq 3.2$

Moderate disease activity (MDA) group: DAS28 $>3.2$ to $\leq 5.1$

High disease activity (HDA) group: DAS28 $>5.1$

\section{Assessment of Anthropometric Parameters and Blood Pressure}

All the participants were informed to report to autonomic function testing (AFT) laboratory maintained at controlled temperature (22$25^{\circ} \mathrm{C}$ ) in the Department of Physiology JIPMER between 9.00 to $11 \mathrm{am}$, two hours after light breakfast. Participants were explained the procedure and precautions to be taken for autonomic function testing during the previous visit. Complete autonomic function test battery was conducted as per European task force guidelines.

Height was measured on a wall mounted stadiometer to the nearest millimetre and weight on a digital weight balanced to the nearest $0.1 \mathrm{~kg}$. BMI was calculated using the formula weight in kilograms divided by square of height in meters. Omron MX3 automated BP monitor (Omron healthcare co, Kyoto, Japan) was used to measure blood pressure after 10 min of rest in supine position.

\section{Assessment of Heart Rate Variability}

The analogue lead-II electrocardiogram (ECG) signals from physiograph was acquired by BIOPAC MP 100 data acquisition system (BIOPAC Inc, USA) and data was screened for the presence of ectopic defects, only normal beats were used for analysis. HRV was analyzed using Kubios HRV software (version 3.0, Bio-signal analysis group, University of Kuopio, Finland). In frequency domain analysis, the power was calculated for very low frequency (VLF, 0.0033-0.04 Hz), low frequency (LF, 0.04-0.15 Hz) and high frequency bands (HF, 0.15-0.40 Hz). Further, total power (TP), low frequency normalized unit (LFnu), high frequency normalized unit (HFnu) and LF-HF ratio (ratio of low frequency power to high frequency power) were recorded. Time domain indices of mean RR (mean-RR interval), RMSSD (square root of the mean squared differences of successive normal to normal intervals), SDNN (standard deviation of normal to normal interval), NN50 (number of interval differences of successive normal to normal intervals greater than 50 milliseconds) and pNN50 (proportion derived by dividing NN50 by the total number of $\mathrm{NN}$ intervals) were recorded, $\mathrm{NN}$-intervals being the intervals between normal R-peaks in ECG. ${ }^{[24]}$

\section{Assessment of Blood inflammatory markers and Cortisol Levels}

Five milliliter of peripheral venous blood was obtained between $7.30 \mathrm{am}$ and 8.30 am for the assessment of inflammatory markers and cortisol by enzyme-linked immunosorbent assay (ELISA) method. Serum was separated by centrifugation after blood sample were allowed to clot for thirty minutes. Serum samples were stored at $-80^{\circ} \mathrm{C}$ until use. The serum IL- 1 alpha, IL- 6 and TNF alpha levels were measured by ELISA kits obtained from Korain Biotech Co Ltd, Shanghai, China. Serum Cortisol levels were measured by the ELISA kits obtained from Calbiotech, Inc (California, USA).

\section{Statistical Analysis of Data}

Using the Kolmogorov-Smirnov test all the data were checked for normality, data found to be non-normally distributed has been expressed as median (interquartile range) and normally distributed as median and standard deviation. Comparison of all the parameters in relation to different disease activity groups has been carried out by Kruskal-Wallis test or ANOVA based on the normality. Partial correlation analysis was used to identify the association between parameters after controlling for the age and disease duration. All statistical analysis was performed at a significance level of 5 percent and the $p<0.05$ was considered as significant. The statistical analysis was performed using SPSS version 19 (SPSS, Chicago, IL, USA).

\section{RESULTS}

A total of 143 patients with RA completed the study. There were 23 participants in LDA group, 69 participants in MDA and 51 in the HDA group (Figure 1). There was no significant difference in age, BMI, Heart rate, systolic and diastolic blood pressure between the three disease activity groups. However, the disease duration was significantly higher in the HDA compared to LDA group (Table 1).

HRV parameters are described in Table 2. LF-HF ratio showed significant increase in MDA group $(p<0.01)$ and HDA group $(p<0.001)$ compared to LDA group (Figure 2). When MDA and HDA groups were compared, it was significantly increased in HDA group as compared to MDA group $(p<0.01)$. Total power was significantly reduced in MDA $(p<0.05)$ 
Ganesan, et al:: Sympathovagal Imbalance in Rheumatoid Arthritis

Table 1: Comparison of age, BMI, blood pressure parameters between LDA, MDA and HDA groups.

$\begin{array}{cccc}\text { Parameters } & \text { LDA }(n=23) & \text { MDA }(n=69) & \text { HDA }(n=51) \\ \text { Age (years) } & 43.7 \pm 8.09 & 41.65 \pm 8.44 & 41.94 \pm 8.44 \\ \begin{array}{c}\text { Gender (Male, } \\ \text { Female) }\end{array} & \text { Male }(n=2) & \text { Male }(n=6) & \text { Male }(n=47) \\ \begin{array}{c}\text { Disease duration } \\ \text { (months) }\end{array} & 12.47 \pm 5.4 & 16.18 \pm 6.92 & 17.17 \pm 6.74^{*} \\ \text { BMI (kg/m²) } & 24.09 \pm 4.62 & 24.39 \pm 3.92 & 24.56 \pm 3.99 \\ \text { HR (per min) } & 82.7 \pm 9.2 & 85.43 \pm 8.24 & 85 \pm 9 \\ \text { SBP (mmHg) } & 116.6 \pm 8.65 & 116.94 \pm 9.62 & 117.24 \pm 9.7 \\ \text { DBP }(\mathrm{mmHg}) & 73.96 \pm 6.44 & 75.42 \pm 7.49 & 74.6 \pm 12.2\end{array}$

Values are expressed as mean \pm standard deviation. BMI: Body mass index; HR: Heart rate; SBP: Systolic blood pressure; DBP: Diastolic blood pressure; LDA: Low disease activity; MDA: Moderate disease activity; HDA: High disease activity. ${ }^{*}$ Mark indicate comparison with LDA group, ${ }^{*} p<0.05 . P<0.05$ is considered to be statistically significant.

Table 2: Comparison of frequency domain indices and time domain indices of HRV between LDA, MDA and HDA groups.

$\begin{array}{cccc}\text { Parameters } & \text { LDA }(n=23) & \text { MDA }(n=69) & \text { HDA }(n=51) \\ \text { TP }\left(\mathrm{ms}^{2}\right) & 447(562) & 417(367)^{*} & 339.3(507)^{*} \\ \text { LFnu } & 56.5(15.7) & 64.1(13.3)^{* *} & 69.9(12.2)^{* * * *} \\ \text { HFnu } & 43.4(16) & 35.8(13.1)^{* *} & 30(12.4)^{* * * *} \\ \text { LF- HF ratio } & 1.3(0.76) & 1.79(1.09)^{* *} & 2.37(1.33)^{* * * *} \\ \text { Mean RR (ms) } & 780(181) & 713.4(144) & 693.2(140) \\ \text { RMSSD (ms) } & 25.6(31) & 15.7(12.1)^{*} & 14.8(17.1)^{* *} \\ \text { SDNN (ms) } & 24.5(22.2) & 18.8(11.2) & 17(17.2)^{*} \\ \text { NN50 } & 13(14) & 8(11.5)^{* *} & 3(10.6)^{* *} \\ \text { pNN50 (\%) } & 2.9(8.4) & 2(3.7) & 0.4(3.9)\end{array}$

Values are expressed as median (interquartile range). TP: Total power; LFnu: Normalized low frequency component; HFnu: Normalized high frequency component; LF-HF ratio: Ratio of the low frequency component to the high frequency component of HRV; Mean RR: Mean-RR interval; RMSSD: Square root of the mean of the sum of the squares of differences between adjacent NN intervals; SDNN: Standard deviation of the averages of NN intervals in all 5 min segments of the entire recording; NN50: Number of interval differences of successive NN intervals greater than $50 \mathrm{~ms}$; pNN50: Proportion derived by dividing NN50 by the total number of NN interval; LDA: Low disease activity; MDA: Moderate disease activity; HDA: High disease activity. ${ }^{*}$ Mark indicate comparison with LDA group and the \# mark indicate comparison with MDA group. ${ }^{*} p<0.05 ;{ }^{* *} p<0.01 ;{ }^{* *} p<0.001 ;{ }^{*} p<0.05 ;{ }^{* *} p<0.01 ;{ }^{* * *} p<0.001 . P<0.05$ is considered to be statistically significant

and HDA group $(p<0.05)$ compared to LDA group, but the difference between MDA and HDA groups was not significant. LFnu was increased in HDA group compared to LDA group $(\mathrm{p}<0.001)$. HFnu was decreased significantly in MDA $(p<0.01)$ and HDA group $(p<0.001)$ compared to LDA group. Time domain parameters of RMSSD, SDNN and NN50 were reduced in HDA group in comparison with LDA group.

Comparison of inflammatory markers between the three disease activity groups is shown in Table 3. Levels of IL-1 $\alpha$ showed significantly higher levels in HDA compared to LDA $(p<0.001)$. Similarly, IL- 6 was increased in HDA compared to LDA $(p<0.05)$ and MDA $(p<0.05)$. Serum cortisol levels were significantly higher in the MDA $(p<0.05)$ and HDA group $(p<0.01)$.
Table 3: Comparison of disease activity, inflammatory markers and cortisol parameters between LDA, MDA and HDA groups.

$\begin{array}{cccc}\text { Parameters } & \text { LDA }(n=23) & \text { MDA }(n=69) & \text { HDA }(n=51) \\ \text { DAS28 } & 2.87(0.32) & 4.72(0.54)^{* * *} & 5.39(0.48)^{* * * * * *} \\ \begin{array}{c}\text { Interleukin } 1 \alpha \\ (\mathrm{pg} / \mathrm{ml})\end{array} & 6.71(10.8) & 8.09(13.2) & 12.5(19.8)^{* * * *} \\ \begin{array}{c}\text { Interleukin } \\ 6(\mathrm{ng} / \mathrm{ml})\end{array} & 93.21(53.8) & 95.6(52.1) & 119.4(63.5)^{* *} \\ \begin{array}{c}\text { TNF- } \alpha(\mathrm{pg} / \mathrm{ml}) \\ \text { Cortisol }(\mathrm{ng} /\end{array} & 78.6(85) & 86.5(79.1) & 97.9(113) \\ \mathrm{mL}) & 84.79(70.2) & 121.1(70.8)^{*} & 144.2(78.9)^{* *}\end{array}$

Values are expressed as Median (Interquartile range). DAS 28: Disease activity score 28; TNF- $\alpha$ : Tumor necrosis factor- $\alpha .{ }^{*}$ Mark indicate comparison with LDA group and the \# mark indicate comparison with MDA group. ${ }^{*} p<0.05$; ${ }^{* *} p<0.01 ;{ }^{* * *} p<0.001 ;{ }^{*} p<0.05 ;{ }^{* *} p<0.01 ;{ }^{* * *} p<0.001 . P<0.05$ is considered to be statistically significant.

Table 4: Correlations of sympathovagal balance with disease activity and inflammatory markers among rheumatoid arthritis patients.

\begin{tabular}{ccc} 
Parameters & \multicolumn{2}{c}{ LF-HF ratio } \\
Disease duration & $r$ & $p$ \\
Disease activity score 28 & 0.131 & 0.12 \\
Interleukin - 1 $\alpha(\mathrm{pg} / \mathrm{ml})$ & 0.473 & 0.000 \\
Interleukin - $6(\mathrm{ng} / \mathrm{L})$ & 0.379 & 0.01 \\
tumor necrosis factor- $\alpha(\mathrm{pg} / \mathrm{ml})$ & 0.092 & 0.272 \\
Cortisol $(\mathrm{ng} / \mathrm{ml})$ & 0.199 & 0.17 \\
\hline
\end{tabular}

LF-HF ratio: Ratio of the low frequency power to the high frequency power. $P<0.05$ is considered to be statistically significant.

Correlation of sympathovagal balance with disease activity and inflammatory markers is shown in Table 4 and Figure 3. Correlation analysis of LF-HF ratio with DAS28 $(r=0.473, p<0.001)$, Cortisol $(\mathrm{r}=0.363, p<0.001), \mathrm{IL}-1 \alpha(\mathrm{r}=0.379, p<0.05)$ showed significant positive association. RMSSD showed weak negative correlation with disease activity score $28(\mathrm{r}=-0.217, \mathrm{p}=0.009)$ and cortisol $(\mathrm{r}=-0.172, \mathrm{p}=0.04)$. SDNN also has weak negative correlation with disease activity score 28 $(\mathrm{r}=-0.205, p=0.04)$ and TNF- $\alpha(\mathrm{r}=-0.183, p=0.04)$.

\section{DISCUSSION}

This study tried to correlate sympathovagal balance with disease activity and inflammatory markers in South Indian patients with newly diagnosed RA.

LF-HF ratio, represents the sympathovagal balance and LFnu, predominately expresses the sympathetic activity, showed significant increase in MDA and HDA group compared to LDA group. Parasympathetic activity represented by HFnu decreased in MDA and HDA groups. Increased LF-HF ratio, LFnu and reduced HFnu indicates that sympathetic activity was increased and parasympathetic activity was decreased in MDA and HDA patients. Evrengul et al. reported sympathovagal imbalance in terms of increased LF-HF ratio, LFnu and reduced HFnu in RA patients compared to healthy controls. ${ }^{[14]}$ Previous study by Yadav et al. found that there was no difference in LF-HF ratio but decreased total power of HRV which represents overall autonomic variability in RA compared to healthy controls. ${ }^{[25]}$ 




Figure 1: Study flow diagram showing the participants flow from screening to analysis.

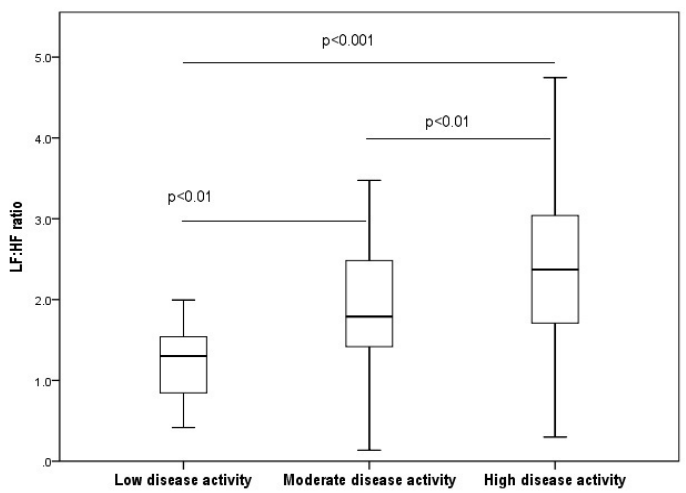

Figure 2: Box plots of LF-HF ratio for LDA, MDA and HDA groups. Box plots of LF-HF ratio (ratio of low frequency power to high frequency power) for LDA, MDA; and HDA groups; LDA: Low disease activity; MDA: Moderate disease activity; HDA: High disease activity. The boxes show the data between the $25^{\text {th }}$ and 75 Percentile; the middle line represents the median. $\mathrm{P}<0.05$ is considered to be statistically significant.

RMSSD and SDNN were decreased in patients with MDA and HDA compared to LDA. Since RMSSD indicates parasympathetic activity and SDNN indicates vagal modulation, results from our study show that the patients with moderate and high medium and high disease activity have reduced parasympathetic activity. A study by Anichkov et al. found that RMSSD and SDNN were lower in RA patients compared to normal controls, suggesting decreased vagal modulation. ${ }^{[26]}$ Jahan et al. also reported reduced RMSSD, SDNN and pNN50\% values in RA patients suggesting autonomic dysfunction. ${ }^{[27]}$

Correlation analysis of LF-HF ratio which represents sympathovagal balance showed moderate positive association with disease activity after controlling for age and disease duration. Therefore, higher the sympathovagal imbalance, higher the disease activity. Particularly, the causality of such an interaction could not be determined by this study and whether a gradual rise in disease activity could result in increased sympathovagal imbalance or excessive sympathovagal imbalance could cause or promote disease activity in RA, remains to be explained. Anichkov et al. reported that disease activity showed negative association with SDNN which represents vagal activity and positive association with disease duration. ${ }^{[26]}$ In our study, there was no correlation between sympathovagal balance and disease duration. Disease duration of 15 months in this study indicates that autonomic dysfunction occurs earlier in rheumatoid arthritis than thought.

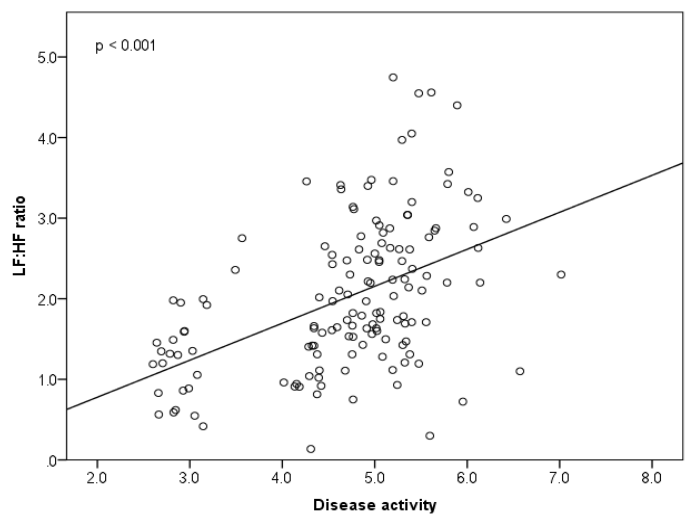

Figure 3: The correlation between LF-HF ratio and disease activity controlling age and disease duration is shown by positive relationship $(r=0.456, p<0.001)$ in the patients with rheumatoid arthritis. LF-HF ratio: Ratio of low frequency power to high frequency power. $P<0.05$ is considered to be statistically significant.

Sympathovagal imbalance is the condition where over activity or under activity of sympathetic or parasympathetic system leads to imbalance of autonomic functions. Commonly, in sympathovagal imbalance, the sympathetic component is more active than the parasympathetic component of ANS. ${ }^{[28,29]}$ Maule et al. demonstrated autoantibodies against sympathetic and parasympathetic nerves in RA subjects compared to normal subjects, which was associated with sympathovagal imbalance. ${ }^{[30]}$ Increased sympathovagal imbalance leads to alteration in circulatory and immune functions. ${ }^{[13]}$ In the present study, weak correlation was found between sympathovagal imbalance and IL$1 \alpha$ and cortisol levels. Previous studies by Czura et al. reported that autonomic nervous system modifies inflammatory marker production via inflammatory reflex. ${ }^{[31]}$ Increased level of sympathovagal imbalance and inflammatory markers may be responsible for the increased cardiovascular risk in RA patients, as altered HRV with increased sympathetic activity and reduced parasympathetic activity are associated with increased cardiovascular risk. ${ }^{[14,15]}$

Several confounding factors can affect HRV, including age, diabetes, hypertension, cardiovascular disease, renal failure, obstructive sleep apnoea, tobacco, caffeine and drugs. ${ }^{[32-34]}$ Therefore, in our study we excluded patients with history of diabetes, hypertension, alcoholism, drug abuse and any condition or drug likely to affect test results. Correlation analysis was done after controlling for age and disease duration. However, some conditions like prediabetes and subclinical cardiovascular disease may affect HRV and single or combination therapy of disease modifying antirheumatic drugs affecting the test results cannot be ruled out.

Strength of this study is a relatively large sample size, inclusion of RA patients with early disease (within three years of disease onset) and inclusion of inflammatory markers. The disease activity correlated with inflammatory markers and HRV. Limitation of this study is that we could not include normal healthy controls, RA patients in remission and those with cardiovascular disease. Autonomic biomarkers like epinephrine, nor-epinephrine and neuropeptide $\mathrm{Y}$ also could not be assessed due to financial constraints. Future longitudinal studies with controls, adequate number of patients in different disease activity groups (including those in remission) and RA patients with cardiovascular disease may help to confirm the role of HRV as a predictor of cardiovascular risk, morbidity and mortality in RA. 
Ganesan, et al:: Sympathovagal Imbalance in Rheumatoid Arthritis

\section{CONCLUSION}

Sympathovagal imbalance was significantly increased in the MDA and HDA group compared to LDA group and it was positively correlated with disease activity, IL-1 $\alpha$ and cortisol in the patients of RA. Assessment of sympathovagal balance may help to find the autonomic dysfunction and cardiovascular risk in newly diagnosed patients with RA.

\section{ACKNOWLEDGMENT}

Authors acknowledge Jawaharlal Institute of Postgraduate Medical Education and Research (JIPMER) for providing financial assistance in the form of Ph.D. Scholarship. We also acknowledge the study participants.

\section{Funding}

Intramural Ph.D. research grant provided by JIPMER, Puducherry (JIP/ Res/Intra-Ph.D/phs1/01/2015-18).

\section{CONFLICT OF INTEREST}

The authors declare that they have no conflict of interest.

\section{ABBREVIATIONS}

RA: Rheumatoid Arthritis; LDA: Low Disease Activity; MDA: Moderate Disease Activity; HAD: High Disease Activity; IL-1 $\alpha$ : Interleukin1a; IL-6: Interleukin-6; TNFa: Tumor Necrosis Factor- $\alpha$; HRV: Heart Rate Variability; AFT: Autonomic Function Testing; ECG: Electrocardiogram; VLF: Very Low Frequency; LF: Low Frequency; HF: High Frequency; RMSSD: Square root of the mean squared differences of successive normal to normal intervals; SDNN: Standard deviation of normal to normal interval; NN50: Number of interval differences of successive normal to normal intervals greater than 50 milliseconds; pNN50: Proportion derived by dividing NN50 by the total number of NN intervals; ELISA: Enzyme-linked immunosorbent assay.

\section{REFERENCES}

1. Mclnnes IB, Schett $G$. The pathogenesis of rheumatoid arthritis. N Engl J Med. 2011;365(23):2205-19.

2. Cojocaru M, Cojocaru IM, Silosi I, Vrabie CD, Tanasescu R. Extra-articular Manifestations in Rheumatoid Arthritis. Maedica. 2010;5(4):286-91.

3. Crowson CS, Liao KP, Davis JM, Solomon DH, Matteson EL, et al. Rheumatoid arthritis and cardiovascular disease. Am Heart J. 2013;166(4):622-8.

4. Fabbri E, An Y, Zoli M, Simonsick EM, Guralnik JM, Bandinelli S, et al. Aging and the burden of multimorbidity: Associations with inflammatory and anabolic hormonal biomarkers. J Gerontol Ser A. 2015;70(1):63-70.

5. Wolfe F, Mitchell DM, Sibley JT, Fries JF, Bloch DA, Williams CA, et al. The mortality of rheumatoid arthritis. Arthritis Rheum. 1994;37(4):481-94

6. Solomon DH, Karlson EW, Rimm EB, Cannuscio CC, Mandl LA, Manson JE, et al. Cardiovascular morbidity and mortality in women diagnosed with rheumatoid arthritis. Circulation. 2003:107(9):1303-7.

7. Holmqvist ME, Wedrén S, Jacobsson LT, Klareskog L, Nyberg F, RantapaaDahlqvist $\mathrm{S}$, et al. Rapid increase in myocardial infarction risk following diagnosis of rheumatoid arthritis amongst patients diagnosed between 1995 and 2006. J Intern Med. 2010;268(6):578-85.

8. DelRincón ID, Williams K, Stern MP, Freeman GL, Escalante A. High incidence of cardiovascular events in a rheumatoid arthritis cohort not explained by traditional cardiac risk factors. Arthritis Rheum. 2001;44(12):2737-45.

9. Sattar N, McCarey DW, Capell H, Mclnnes IB. Explaining how "high-grade" systemic inflammation accelerates vascular risk in rheumatoid arthritis. Circula- tion. 2003;108(24):2957-63.

10. McCraty R, Shaffer F. Heart Rate Variability: New Perspectives on Physiological Mechanisms, Assessment of Self-regulatory Capacity and Health risk. Glob Adv Health Med. 2015;4(1):46-61.

11. Pagani M, Lombardi F, Guzzetti S, Rimoldi O, Furlan R, Pizzinelli $P$, et al. Power spectral analysis of heart rate and arterial pressure variabilities as a marker of sympatho-vagal interaction in man and conscious dog. Circ Res. 1986;59(2):17893.

12. Malliani A, Pagani M, Lombardi F, Cerutti S. Cardiovascular neural regulation explored in the frequency domain. Circulation. 1991;84(2):482-92.

13. Pal GK. Sympathetic and parasympathetic systems. In: Text book of Medical Physiology. $1^{\text {st }}$ ed. New Delhi: Jaypee Brother's Medical Publications. 2016;295 311

14. Evrengül H, Dursunoglu D, Cobankara V, Polat B, Seleci D, Kabukcu S, et al Heart rate variability in patients with rheumatoid arthritis. Rheumatol Int. 2004;24(4):198-202

15. LaRovere MT, Pinna GD, Maestri R, Mortara A, Capomolla S, Febo O, et al. Short-term heart rate variability strongly predicts sudden cardiac death in chronic heart failure patients. Circulation. 2003;107(4):565-70.

16. Sattar N, Mclnnes IB. Vascular comorbidity in rheumatoid arthritis: Potential mechanisms and solutions. Curr Opin Rheumatol. 2005;17(3):286-92.

17. Lazzerini PE, Capecchi PL, Laghi-Pasini F. Systemic inflammation and arrhythmic risk: Lessons from rheumatoid arthritis. Eur Heart J. 2017;38(22):1717-27.

18. Li YH, Rozanski GJ. Effects of human recombinant interleukin-1 on electrical properties of guinea pig ventricular cells. Cardiovasc Res. 1993;27(3):525-30.

19. Wang J, Wang H, Zhang $Y$, Gao H, Nattel S, Wang Z. Impairment of HERG K(+) channel function by tumor necrosis factor-alpha: Role of reactive oxygen species as a mediator. J Biol Chem. 2004:279(14):13289-92.

20. Hagiwara Y, Miyoshi S, Fukuda K, Nishiyama N, Ikegami Y, Tanimoto K, et al SHP2-mediated signaling cascade through gp130 is essential for LIF-dependent I CaL, [ $\mathrm{Ca}_{2+1}$ i transient and APD increase in cardiomyocytes. J Mol Cell Cardiol. 2007;43(6):710-6

21. Pal GK. Association of cardiovascular risks with sympathovagal imbalance in rheumatoid arthritis. Indian J Med Res. 2012;136(4):547-8.

22. Aletaha D, Neogi T, Silman AJ, Funovits J, Felson DT, Bingham CO, et al. 2010 rheumatoid arthritis classification criteria: An American College of Rheumatology/European League Against Rheumatism collaborative initiative. Ann Rheum Dis. 2010;62(9):1580-8.

23. Anderson J, Caplan L, Yazdany J, Robbins ML, Neogi T, Michaud K, et al. Rheumatoid arthritis disease activity measures: American College of Rheumatology recommendations for use in clinical practice. Arthritis Care Res. 2012:64(5):6407.

24. Shaffer F Ginsberg JP. An Overview of Heart Rate Variability Metrics and Norms. Front Public Health. 2017:5:258.

25. Yadav RK, Gupta R, Deepak KK. A pilot study on short term heart rate variability and its correlation with disease activity in Indian patients with rheumatoid arthritis. Indian J Med Res. 2012;136(4):593-8.

26. Anichkov DA, Shostak NA, Ivanov DS. Heart rate variability is related to disease activity and smoking in rheumatoid arthritis patients. Int J Clin Pract. 2007;61(5):777-83.

27. Jahan K, Begum N, Ferdousi S. Heart Rate Variability in Patients with Rheumatoid Arthritis. Journal of Bangladesh Society of Physiologist. 2013;7(2):78-82

28. Pal GK. Role of sympathovagal balance in achieving effective homeostasis. Biomedicine. 2008;28:67-8

29. Ondicova K, Mravec B. Multilevel interactions between the sympathetic and parasympathetic nervous systems: A minireview. Endocr Regul. 2010;44(2):69-75

30. Maule S, Quadri R, Mirante D, et al. Autonomic nervous dysfunction in systemic lupus erythematosus (SLE) and rheumatoid arthritis (RA): Possible pathogenic role of autoantibodies to autonomic nervous structures. Clin Exp Immunol. 1997;110(3):423-7.

31. Czura CJ, Tracey KJ. Autonomic neural regulation of immunity. J Intern Med 2005;257(2):156-66

32. Dinas PC, Koutedakis Y, Flouris AD. Effects of active and passive tobacco cigarette smoking on heart rate variability. Int J Cardiol. 2013;163(2):109-15.

33. Rajendra AU, Paul JK, Kannathal N, Lim CM, Suri JS. Heart rate variability: A review. Med Biol Eng Comput. 2006;44(12):1031-51.

34. Karapetian GK, Engels HJ, Gretebeck KA, Gretebeck RJ. Effect of caffeine on LT, VT and HRVT. Int J Sports Med. 2012;33(7):507-13 